\title{
Enhancing memory performance with rTMS in healthy subjects and individuals with Mild Cognitive Impairment: the role of the right dorsolateral prefrontal cortex
}

\author{
Patrizia Turriziani ${ }^{1 *}$, Daniela Smirni ${ }^{1}$, Giuseppe Zappalà ${ }^{4}$, Giuseppa R. Mangano ${ }^{1}$, \\ Massimiliano Oliveri ${ }^{1,3}$ and Lisa Cipolotti ${ }^{1,2}$ \\ 1 Dipartimento di Psicologia, Università di Palermo, Palermo, Italy \\ ${ }^{2}$ Department of Neuropsychology, National Hospital for Neurology and Neurosurgery, Queen Square, London, UK \\ 3 IRCCS Fondazione "Santa Lucia," Roma, Italy \\ ${ }^{4}$ Unità di Neurologia Cognitiva e Riabilitazione, ARNAS Garibaldi, Catania, Italy
}

Edited by:

Simone Rossi, Azienda Ospedaliera

Universitaria Senese, Italy

Reviewed by:

Maria Cotelli, IRCCS Centro San

Giovanni di Dio - Fatebenefratelli,

Italy

David Bartrés-Faz, University of

Barcelona, Spain

*Correspondence:

Patrizia Turriziani, Dipartimento di Psicologia, Università di Palermo,

Viale delle Scienze, Ed. 15, Palermo 90128, Italy.

e-mail: patrizia.turriziani@unipa.it
A debated question in the literature is the degree of anatomical and functional lateralization of the executive control processes sub-served by the dorsolateral prefrontal cortex (DLPFC) during recognition memory retrieval. We investigated if transient inhibition and excitation of the left and right DLPFC at retrieval by means of repetitive transcranial magnetic stimulation (rTMS) modulate recognition memory performance in 100 healthy controls (HCs) and in eight patients with Mild Cognitive Impairment (MCl). Recognition memory tasks of faces, buildings, and words were used in different experiments. rTMS-inhibition of the right DLPFC enhanced recognition memory in both HCs and MCls. rTMS-excitation of the same region in HCs deteriorated memory performance. Inhibition of the right DLPFC could modulate the excitability of a network of brain regions, in the ipsilateral as well as in the contralateral hemisphere, enhancing function in $\mathrm{HCs}$ or restoring an adaptive equilibrium in $\mathrm{MCl}$.

Keywords: rTMS, DLPFC, MCI, recognition memory, retrieval

\section{INTRODUCTION}

The role of the prefrontal cortex (PFC) in recognition memory retrieval has been well documented (e.g., Simons and Spiers, 2003; Squire, 2004). It remains an open question as to the degree of anatomical and functional lateralization of the PFC control processes involved in retrieval.

Lesion (e.g., Milner et al., 1991), neuroimaging (e.g., Wagner et al., 1998; McDermott et al., 1999), and TMS (e.g., Turriziani et al., 2008, 2010) studies have reported material specific laterality effects in the activation of PFC during recognition memory, showing that the left and right PFC are differentially recruited, respectively, for verbal and non-verbal memoranda.

Other neuroimaging and TMS studies have reported different patterns of activation of PFC areas depending on the processes of encoding and retrieval in recognition memory of both verbal and non-verbal memoranda (Kirchhoff et al., 2000; Buckner and Wheeler, 2001; Fletcher and Henson, 2001; Rossi et al., 2001; Sandrini et al., 2003; Floel et al., 2004). The HERA modelHemispheric Encoding Retrieval Asymmetry (e.g., Tulving et al., 1994; Habib et al., 2003) suggests that the left PFC plays a crucial role in encoding, whereas right PFC is necessary for retrieval (but see Spaniol et al., 2009). This pattern of lateralization can be influenced by the use of retrieval strategies. For example, a recent study showed that during memory retrieval the right DLPFC is preferentially recruited if memory strategies are used to guide episodic retrieval, whereas when the subject does not apply a retrieval strategy there is a shift to the left DLPFC (Manenti et al., 2010).

A different pattern of lateralization of PFC can also be observed during aging. In normal aging, PFC activations tend to be less asymmetric during memory tasks, as indicated by the HAROLD model [Hemispheric Asymmetry Reduction in Older adults (Cabeza, 2002)]. Asymmetry reductions could reflect a compensation mechanism, helping to counteract the age-related cognitive decline, or a de-differentiation process, reflecting the age-related failure to allocate functional resources in a selective manner. Most available evidence, tend to support the compensation hypothesis. Brain stimulation studies, for example, have shown that high frequency rTMS trains, when applied on-line during encoding or retrieval of memory traces, exert a different pattern of interference on memory encoding and retrieval depending on subjects' age. Rossi et al. (2004), using an highfrequency on-line rTMS approach, reported that in young subjects, rTMS of the right DLPFC interfered with retrieval more than left DLPFC stimulation. This asymmetry progressively vanished with aging, as indicated by bilateral interference effects on recognition performance.

Manenti et al. (2011) used the same on-line TMS approach to transiently interfere with the function of the dorsolateral prefrontal cortex (DLPFC) during encoding or retrieval of associated and non-associated word pairs in high- and low-performing older adults. The predominance of left vs. right DLPFC effects during 
encoding, predicted by the HERA model, was observed only in low-performing older adults, while the asymmetry reduction predicted by the HAROLD model was selectively shown for the high-performing group.

Solé-Padullés et al. (2006), using off-line high-frequency rTMS to increase cortical excitability of the left and right DLPFC before memory tasks, improved learning of face-name associations in elderly participants with memory dysfunction, an effect that was paralleled by increased metabolic activation of the right DLPFC (Solé-Padullés et al., 2006).

Consistently with these data, bilateral recruitment of DLPFC has been shown to facilitate performance in physiological as well as in pathological brain aging (e.g., Cabeza et al., 2002; Rosen et al., 2002). Neuroimaging studies documented increased activation in the right DLPFC associated with memory deficits in MCI and AD (e.g., Wang et al., 2006; Bai et al., 2009). Some studies interpreted the increased right DLPFC recruitment as recruitment of compensatory networks (e.g., Grady et al., 2003; Smith et al., 2007), while other studies (e.g., Grady et al., 2001) suggested that activity levels in the right DLPFC are negatively correlated with memory performance. These results suggest that at least for certain tasks, bilateral DLPFC recruitment may reflect maladaptive plasticity processes rather than compensatory ones.

To date, only one study investigated whether rTMS modulates memory in neurological patients. Cotelli et al. (2011) reported that high-frequency rTMS of the left parietal cortex increased accuracy in an association memory task in a patient with amnestic mild cognitive impairment (MCI), and this improvement was still significant 24 weeks after stimulation began.

In the present study, we investigated if transient inhibition and excitation of the DLPFC at retrieval by means of rTMS can modulate recognition memory performance in healthy subjects and in MCI patients. First, we explored whether left and right DLPFC are differentially involved in the retrieval processes of verbal and non-verbal memoranda. Secondly, we explored how temporary inhibition and excitation of the DLPFC may modulate performance on forced-choice recognition memory tasks. Third, we explored whether temporary inhibition and excitation of the right DLPFC is detrimental or helpful in forced-choice recognition memory tasks in healthy controls (HCs) and in patients with MCI.

\section{EXPERIMENTAL INVESTIGATION PARTICIPANTS Healthy subjects}

One hundred participants (78 females, age-range 20-35 years; education 10-12 years), were recruited from a predominantly female students' population. All participants were right-handed, native Italian speakers, with no previous history of neurological or psychiatric problems. Informed consent was obtained from all subjects in accordance with the independent Ethics Committee of the Foundation IRCCS Santa Lucia of Rome.

\section{$\mathrm{MCl}$}

Eight MCI patients (six males) were recruited from patients who, following memory complaints, consulted a local neurologically lead cognitive disorder clinic. All patients met the diagnostic criteria for MCI (Petersen et al., 1999). They all had: (1) subjective memory impairment (over 1-2 years); (2) objective poor memory performance as assessed by the California Verbal Learning test (Delis et al., 1988) and the Rey Complex figure (Carlesimo et al., 2002); (3) normal general cognitive functioning as assessed by the Raven's Colored Progressive Matrices (Carlesimo et al., 1996) and tests of short-term memory, naming, and frontal "executive" functions; (4) a Clinical Dementia Rating score below 0.5 (Hughes et al., 1982); (5) no or minimal impairment in activities of daily living (ADL) as assessed by the Instrumental Activities of Daily Living scale (IADL) and by the ADL scale; (6) absence of dementia [score > 24 on MMSE (Folstein et al., 1975); Table 1]. All patients were free of medications. The MCI individuals exhibited no history of other neurological or psychiatric disorders, alcohol abuse, psychosis, or major depression. Neurological investigation was normal in all eight patients.

\section{MATERIALS}

The materials used have been employed in previous studies where they have been described in detail (Turriziani et al., 2010); a Italian version (Smirni et al., 2010) of Recognition Memory Test (Warrington, 1984, 1996). Therefore, we will only provide a brief outline. The stimuli used in the non-verbal recognition memory tasks were either unknown faces (Faces Recognition) or unknown buildings (Buildings Recognition). The faces were black and white photographs of Caucasian

Table 1 | MCl patients' demographic data and cognitive scores.

\begin{tabular}{lll}
\hline & MCl patients & Maximum possible score \\
\hline Age (years) & $66.4(5.7)$ & \\
Education (years) & $13.6(3.7)$ & \\
CDR & $0.2(0.3)$ & 5 \\
ADL & $6(0)$ & 6 \\
IADL & $7.6(0.5)$ & 8 \\
MMSE & $26.9(2)$ & 30 \\
GDS & $3.4(2.3)$ & 15 \\
- Delayed Rey's figure & $4.2^{*}(2.9)$ & 36 \\
- CVLT & $28.6^{*}(6.3)$ & 80 \\
- CRPM & $27.5(3.7)$ & 36 \\
- Digit span & $4.9(1.3)$ & \\
- Corsi & $4.9(1)$ & \\
- BNT & $51.2(3.9)$ & 60 \\
$\dagger$ & $9(2.1)$ & \\
- FAS & $17.8(3.9)$ & \\
\hline
\end{tabular}

* Indicates a pathological score (below the lower limit of $95 \%$ tolerance interval measured in the normal population).

Legend: $\bullet=x^{-}$number correct responses; standard deviation values in brackets. ${ }^{\dagger}=$ number of correct responses produced in $1 \mathrm{~min} ;=R T$.

CDR, Clinical Dementia Rating scale; ADL, Activities daily living; IADL, Instrumental Activities daily living; MMSE, Mini Mental State Examination; GDS, Geriatric Depression Scale; CRPM, Colored Raven's Progressive Matrices; CVLT, California Verbal Learning Test (total words recalled trials 1-5); BNT, Boston Naming Test; FAS, Category phonemic fluency (F letter). 
women, approximately 25-years old, with Italian physiognomic characteristics, neutral expression, and no obvious distinguishing features. The buildings were black and white photographs of houses or palaces with Italian architectonical characteristics, neutral style, no obvious distinguishing features and with verbal cues digitally removed (e.g., house numbers, street names, etc.).

The stimuli used in the verbal recognition memory tasks were two sets of concrete and abstract words (set A and set B) adapted from Laudanna et al. (1995), written in capital letters, 4-6 letters long, comparable in frequency (mean 5.91 \pm 5.89 ).

\section{PROCEDURE}

In the computerized tasks, in the study phase, 30 study stimuli were presented individually, in the center of a computer screen over a white background. The non-verbal stimuli (faces and buildings) were presented for $500 \mathrm{~ms}$ whilst the verbal stimuli were presented for $250 \mathrm{~ms}$. This shorter presentation rate for the verbal stimuli was used to ensure similar level of performance in the verbal and non-verbal recognition tasks. The stimuli were preceded by a fixation point lasting $500 \mathrm{~ms}$. The inter-stimulus interval (ISI) was $3500 \mathrm{~ms}$. Each target stimulus was presented in the center of a computer screen over a white background.

Participants were instructed to judge whether the stimulus presented in the study phase was "pleasant" or "unpleasant." This judgment task has been previously used (Cipolotti et al., 2006, 2008; Turriziani et al., 2010) to focus subjects' attention in stimulus encoding. Participants responded by pressing one of the two designated keys on the keyboard.

The recognition phase was administered after an interval of $10 \mathrm{~min}$. In the recognition phase, a three alternative forced choice recognition memory task was administered. Thirty stimulus triplets were presented. In each triplet, the target was presented with two other similar distractors, vertically arranged. The target was presented in a balanced order either in the upper, lower, or middle quadrant. For the Faces task, the two distractors were two faces with physiognomic characteristic similar to the target. This similarity was established in a pilot study, in which participants were asked to judge the face similarity on the basis of hair and color configuration, eyes color and shape, nose and mouth shape. For the Buildings task, the two distractors belonged to the same subcategory (for example, target houses were paired with architecturally similar distractors houses, target palaces were paired with architectonically similar distractors palaces). For the Words task, the two distractors were words semantically or phonologically similar to the target (e.g., melon, pineapple, watermelon).

The recognition trial began with a fixation point of $500 \mathrm{~ms}$ followed by the presentation of the triplets (target and two distractors) for $2 \mathrm{~s}$. The ISI was $3500 \mathrm{~ms}$. The duration of the recognition phase was identical for the non-verbal and verbal tests (see Figure 1 for an example). Subjects were asked to recognize the previously presented stimuli by pressing one of three response buttons vertically arranged on the keyboard. If unsure they were asked to guess.

Responses were measured in terms of accuracy and reaction times (RTs). Accuracy was the number of correct targets

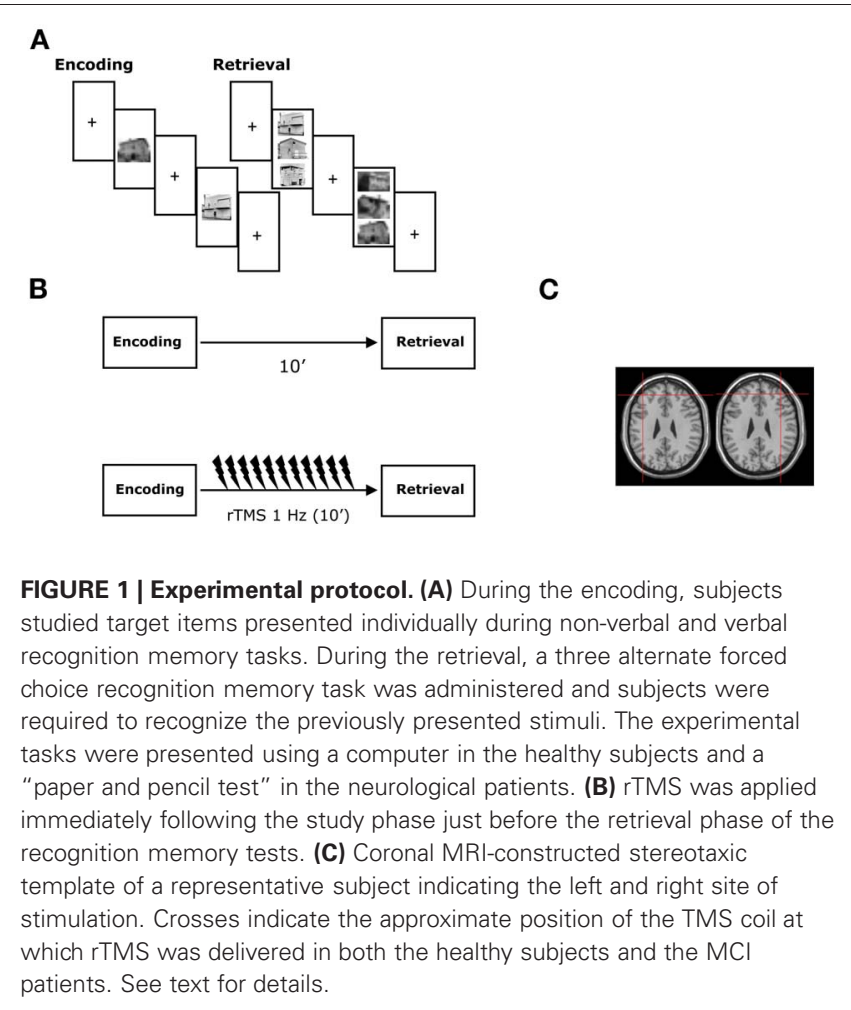

that participants were able to identify in the three forcedchoice recognition memory test. The RTs were considered as the time interval from the onset of the test stimuli to the subject's response.

A paper and pencil version of the non-verbal recognition memory task was used in the MCI patients in the attempt to mach their performance to that of the HCs. In this task, in the study phase, each stimulus was presented individually by the examiner on a white A4 sheet for $3 \mathrm{~s}$. The patient was asked to judge whether the stimulus was pleasant or not. In the recognition phase, the examiner presented triplets of stimuli comprising the target stimulus and two distractors in individual white A4 sheets. The patients were asked to point to the stimulus they thought they had seen before. If unsure, patients were asked to guess. When the patient responded, the examiner presented the next triplet. The duration of the recognition phase was approximately $2-3 \mathrm{~min}$. This is an interval of time well within the expected TMS conditioning after-effects (Di Lazzaro et al., 2011). Only accuracy was recorded in this task.

\section{rTMS}

rTMS was delivered by means of a MagStim Super-Rapid magnetic stimulator, using a figure-of-eight coil (70 $\mathrm{mm}$ in diameter). In all participants, rTMS was applied over the left and right DLPFC, following the study phase, before recognition phase. According to previous studies guidelines for stimulation of the DLPFC, the tip of the intersection of the two coil loops was lined up with the F3/F4 sites of the 10-20 EEG system. The Tailarach coordinates of the stimulated cortical site were automatically estimated in a representative subject from an MRI 
constructed stereotaxic template using Softaxic software. They corresponded approximately at $\pm 40,45,28$ (Turriziani et al., 2010).

In Experiments 1, 2, and 4, inhibitory rTMS was applied, using trains of 600 stimuli at $1 \mathrm{~Hz}$ frequency and at an intensity of $90 \%$ of the motor threshold (MT). MT was defined as the lowest TMS intensity (as assessed with single-pulse TMS) able to induce a visible muscle twitch of the contralateral hand in at least $50 \%$ of a sequence of 10 consecutive trials (Rossini et al., 1994). MT was determined on the same hemisphere of the stimulated left and right DLPFC. There were no interhemispheric differences in MT values both in HCs (left hemisphere: $64 \pm 7.6 \%$; right hemisphere: $64.6 \pm 5.8 \%$ ) and in MCI patients (left hemisphere: $60 \pm 7.0 \%$; right hemisphere: $60.7 \pm 8.9 \%$ ). There was no significant difference between MT values of HCs and MCI patients.

In Experiment 3, excitatory rTMS trains were applied, using the intermittent theta burst stimulation (iTBS) protocol (Huang et al., 2005). Bursts consisting of three pulses at $50 \mathrm{~Hz}$, at an intensity of $80 \%$ aMT, were repeated every $200 \mathrm{~ms}$ (i.e., $5 \mathrm{~Hz}$ ) for $2 \mathrm{~s}$. This 2-s train was repeated once every $10 \mathrm{~s}$ for 20 repetitions, a total of $193 \mathrm{~s}$ (i.e., 600 stimuli). This stimulation protocol has been shown to produce an increase in corticospinal excitability lasting up to $20 \mathrm{~min}$ (Huang et al., 2005). To evaluate active motor threshold (aMT), electromyographic recordings were made using a belly-to-tendon montage from the left first dorsal interosseous (FDI) muscle. The raw signal was amplified and filtered with a band-pass filter of $30 \mathrm{~Hz}-1 \mathrm{kHz}$ (Digitimer Ltd.), and signals were digitized at $2 \mathrm{kHz}$ (CED Power1401, Cambridge Electronic Design). The location of the hand representation in the right hemisphere was determined, defined as the position at which stimulation produced optimal MEPs in the left FDI. The aMT was assessed during voluntary contraction of the target FDI at $\sim 10 \%$ of maximum force and was defined as the lowest stimulus intensity required to evoke an MEP of $>200 \mu \mathrm{V}$ in 5 of 10 trials.

In all experiments, sham rTMS blocks were conducted, with the coil held close to the DLPFC but angled away.

\section{STATISTICAL ANALYSIS}

HCs data were analyzed with an ANOVA for repeated measures, with Side (left, right) as between-subjects factor and Condition (sham rTMS, rTMS) as within-subjects factor. MCIs data were analyzed with an ANOVA for repeated measures with Side (left, right) and Condition (sham rTMS, rTMS) as within-subjects factors. Planned comparisons of single factors were carried out only when significant group factors were found.

\section{EXPERIMENT 1: 1 HZ rTMS OF THE DLPFC. NON-VERBAL RECOGNITION MEMORY}

Forty participants were randomly allocated into a left DLPFC group (20 subjects), receiving sham and real rTMS over the left DLPFC, or a right DLPFC group (20 subjects), receiving sham and real rTMS over the right DLPFC. Sham and real rTMS were given in two separate sessions in the same day, separated by a $6 \mathrm{~h}$ delay. In each condition (sham rTMS or real rTMS), the subjects were administered either the Faces recognition or
Buildings recognition tests. Administration of these two tasks in sham or real rTMS conditions was counterbalanced across subjects.

\section{EXPERIMENT 2: 1 HZ rTMS OF THE DLPFC. VERBAL RECOGNITION MEMORY}

Forty participants different from those recruited for Experiment 1 were recruited and randomly allocated into a left DLPFC group (20 subjects), or a right DLPFC group (20 subjects).

A verbal recognition memory task was administered. Two sets of words (set A and set B) were used either during sham and rTMS conditions. The same procedure as in Experiment 1 was used.

\section{EXPERIMENT 3: ITBS OF THE DLPFC. NON-VERBAL RECOGNITION MEMORY}

Twenty participants were recruited and randomly allocated into a left DLPFC group (10 subjects), or a right DLPFC group (10 subjects). The same stimuli and procedure as in Experiment 1 were used.

\section{EXPERIMENT 4: 1 HZ rTMS OF THE DLPFC. NON-VERBAL RECOGNITION MEMORY IN MCI PATIENTS}

One $\mathrm{Hz}$ rTMS was applied to the left and right DLPFC of MCI patients performing non-verbal recognition memory tasks. Each patient received sham and real rTMS over the left DLPFC (Session 1) and over the right DLPFC (Session 2). The two sessions were separated by an interval of approximately three weeks. Sham and real rTMS were given in the same day, $6 \mathrm{~h}$ apart. The order of sham and real rTMS was counterbalanced across subjects. The two versions of the non-verbal recognition memory task (Face Recognition and Building Recognition) were assigned, counterbalanced across subjects, to rTMS or sham rTMS conditions.

\section{RESULTS}

\section{EXPERIMENT 1: 1 HZ rTMS OF THE DLPFC—NON-VERBAL RECOGNITION MEMORY \\ Accuracy}

Side $\left[F_{(1,38)}=3.32 ; p<0.07\right]$ and Condition $\left[F_{(1,38)}=1.85\right.$; $p<0.18$ ] effects were not significant. The Side $\times$ Condition interaction was significant $\left[F_{(1,38)}=5.27 ; \quad p<0.02\right]$ (Figure 2A). Right rTMS significantly improved subjects' accuracy when compared with sham rTMS $\left[F_{(1,38)}=6.69\right.$; $p<0.01]$. There was no significant difference in subjects' accuracy between left rTMS and sham rTMS $\left[F_{(1,38)}=0.43\right.$; $p<0.51]$. There was no significant difference between left and right sham rTMS conditions $\left[F_{(1,38)}=0.001 ; p<0.97\right]$.

\section{RTs}

The ANOVA performed on RTs showed no significant effects (Side: $F_{(1,38)}=0.09 ; p<0.76$; Condition. $F_{(1,38)}=0.07 ; p<$ 0.78 ; Side $\times$ Condition: $\left.F_{(1,38)}=1.11 ; p<0.29\right)$.

Our results indicate that rTMS over the right DLPFC significantly improves non-verbal recognition memory performance without any significant modulation of speed of response. 


\section{EXPERIMENT 2: 1 HZ rTMS OF THE DLPFC. VERBAL RECOGNITION MEMORY \\ Accuracy}

No significant main effects of Side $\left[F_{(1,38)}=1.61 ; p<0.21\right]$ or Condition $\left[F_{(1,38)}=1.70 ; p<0.19\right]$ were found. The Side $\times$ Condition interaction was significant $\left[F_{(1,40)}=5.79 ; p<0.02\right]$ (Figure 2B). Right rTMS significantly improved subjects' accuracy when compared with sham rTMS $\left[F_{(1,40)}=6.88 ; p<0.01\right]$. Left rTMS did not affect accuracy $\left[F_{(1,40)}=0.60 ; p<0.44\right]$. There was no significant difference between the left and right sham rTMS conditions $\left[F_{(1,38)}=0.005 ; p<0.94\right]$.

\section{RTs}

The ANOVA performed on the RT data did not reveal any significant effect.

These results indicate that rTMS over the right DLPFC significantly improves verbal recognition memory performance, without any significant modulation of speed of response. These results, in conjunction with those obtained in the previous experiment, suggest that right rTMS before recognition improves the healthy subjects' recognition memory accuracy, regardless of the type of memoranda.

\section{EXPERIMENT 3: iTBS OF THE DLPFC. NON-VERBAL RECOGNITION MEMORY \\ Accuracy}

The Side effect was not significant $\left[F_{(1,18)}=1.86 ; p>0.18\right]$. Condition $\left[F_{(1,18)}=8.81 ; p<0.008\right]$ and Side $\times$ Condition interaction $\left[F_{(1,18)}=11.61 ; p<0.003\right]$ were significant. Right iTBS significantly impaired non-verbal recognition accuracy when compared with sham iTBS $\left[F_{(1,18)}=20.31 ; p<0.0002\right]$. In contrast, there was no significant difference in subjects' accuracy after left iTBS compared with sham iTBS $\left[F_{(1,18)}=0.9\right.$; $p<0.759$; Figure 3]. There was no significant difference between the left and right sham iTBS conditions $\left[F_{(1,18)}=0.5 ; p<0.48\right]$.

\section{RTs}

No significant effects of Side $\left[F_{(1,18)}=0.2001 ; p<0.66\right]$, Condition $\left[F_{(1,18)}=0.0023 ; p<0.9626\right]$ and Side $\times$ Condition interaction $\left[F_{(1,18)}=0.0047 ; p<0.9461\right]$ were found.

Our results indicate that iTBS over the right DLPFC in HCs significantly impairs performance on the non-verbal recognition

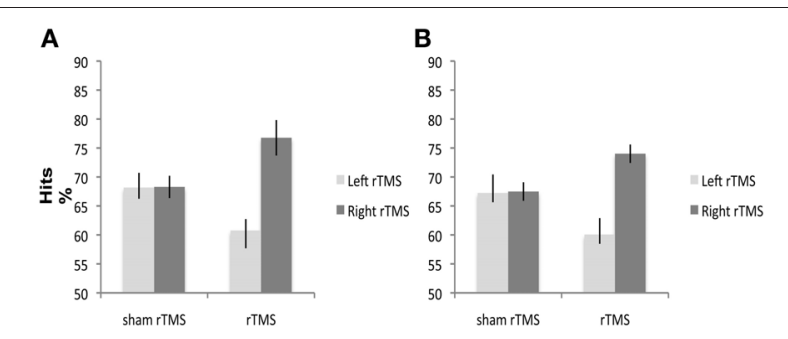

FIGURE 2 | A and B. Experiment 1 and 2: $1 \mathrm{~Hz}$ rTMS over right and left DLPFC has different effects on mean percentage of healthy subjects' correct responses. Right rTMS significantly improves performance of healthy subjects' performance on (A) non-verbal and (B) verbal recognition memory tasks. Square symbols represent individuals under right DLPFC, diamond symbols individuals under left DLPFC. memory task without any significant modulation of speed of response.

\section{EXPERIMENT 4: 1 HZ rTMS OF THE DLPFC. NON-VERBAL RECOGNITION MEMORY IN MCI PATIENTS \\ Accuracy}

The Side effect was not significant $\left[F_{(1,7)}=4.09 ; p>0.18\right]$. Condition $\left[F_{(1,7)}=12.46 ; p<0.009\right]$ and Side $\times$ Condition interaction $\left[F_{(1,7)}=15.89 ; p<0.05\right]$ were significant. Right rTMS $\left[F_{(1,7)}=18.66 ; p<0.003\right]$ improved non-verbal recognition memory performance in all eight patients when compared with sham rTMS. Left rTMS again did not affect accuracy $\left[F_{(1,7)}=2.02 ; p<0.19\right]$ (Figure 4). There was no significant difference between the left and right sham rTMS conditions $\left[F_{(1,7)}=1.74 ; p<0.22\right]$.

These findings replicated and extended the results obtained in HCs in Experiment 1. In the MCI patients, just as in the HCs, non-verbal recognition memory accuracy significantly improved following rTMS over right but not left DLPFC.

\section{DISCUSSION}

This study investigated the effects of rTMS over left and right DLPFC at retrieval in verbal and non-verbal recognition memory tests. We used inhibitory and excitatory rTMS paradigms in HCs and in MCIs. rTMS-inhibition of the right DLPFC improved recognition memory performance in HCs for both verbal and
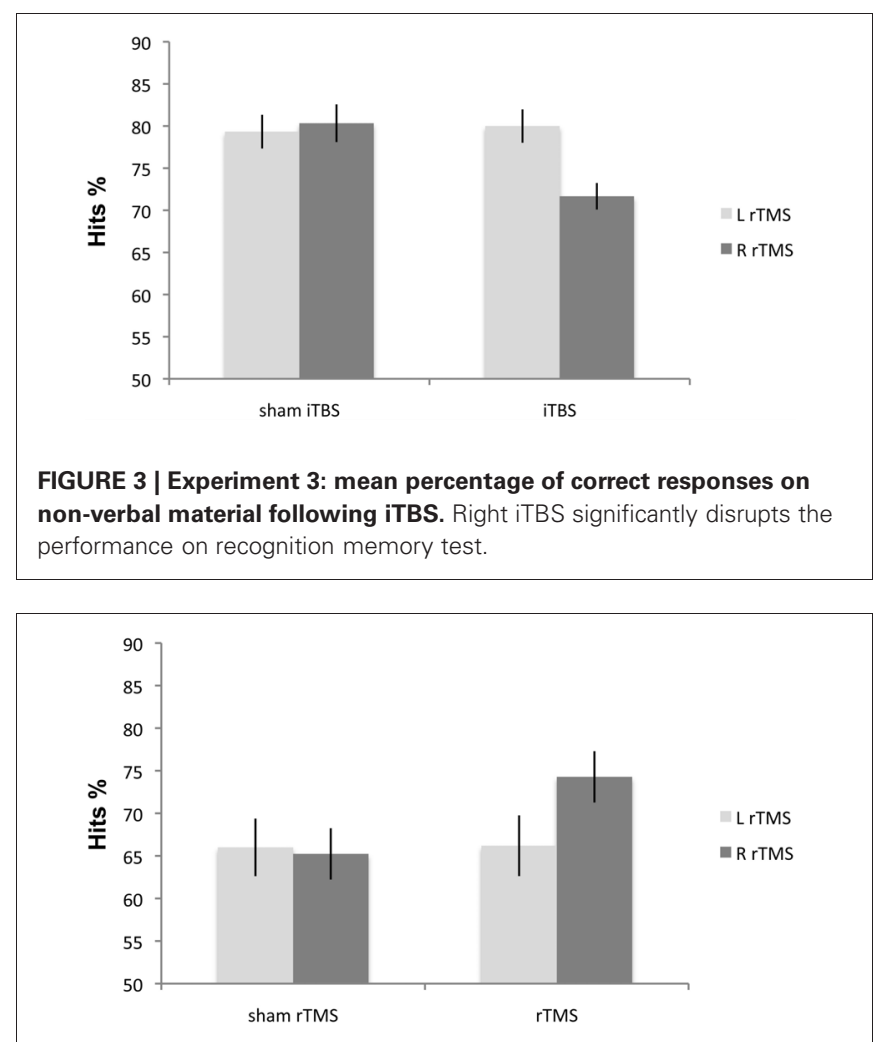

FIGURE 4 | Experiment 4: mean percentage correct responses of MCI patients on the non-verbal recognition memory task. Right rTMS significantly improves the performance on recognition memory test. 
non-verbal memoranda. rTMS-inhibition of the left DLPFC had no effect in the recognition memory performance in the HCs. However, iTBS-excitation of the right DLPFC of HCs impaired non-verbal recognition memory performance. iTBS-excitation of the left DLPFC had no effect on recognition memory performance.

Remarkably, rTMS-inhibition of the right DLPFC also improved the recognition memory performance of MCI patients with memory deficits. In contrast and similarly to the HCs, the performance of the MCI did not improve following inhibitory rTMS on the left DLPFC.

As far as we are aware, this is the first documentation of improvement in recognition memory performance following application of inhibitory rTMS. Remarkably, this improvement is specific to inhibition of the right DLPFC and it occurs for both verbal and non-verbal memoranda in HC. This finding suggests that inhibition of the right DLPFC modulates a domain-general memory retrieval process (Rossi et al., 2001; Sandrini et al., 2003). However, these results are somewhat difficult to reconcile with data from lesions and neuroimaging studies reporting that left and right PFC are differentially involved in verbal and non-verbal memory tasks (e.g., Milner et al., 1991; Wagner et al., 1998). A recent study showed that rTMS over left and right DLPFC induces material specific laterality effects in recognition memory tasks tapping recollection and familiarity at encoding but not at retrieval (Turriziani et al., 2010). All these findings could reconcile material specific and process specific hypothesis of DLPFC recruitment in recognition memory, suggesting that material-specific laterality effects may emerge at encoding but not at retrieval, while a right hemispheric lateralization of DLPFC would be associated specifically with retrieval.

However, since rTMS was applied before the retrieval phases, it difficult to exclude unequivocally that right rTMS could have modulated a number of operations occurring in post-encoding neurocognitive processing (e.g., van Kesteren et al., 2010). The present experiments do not allow demonstrating specifically which process was responsible for the memory improvement observed. Different experimental paradigms targeting different phases of post-encoding will better address this issue.

Previous studies performing similar designs (e.g., Rossi et al., 2001; Manenti et al., 2010) used on-line stimulation during encoding/retrieval, allowing a better neurochronometric alignment between interference in a precise moment and behavioral outcome. On the other hand, off-line TMS as employed in this study allows studying plastic effects of stimulation at brain sites distant from those stimulated. We speculate that if the effects of rTMS were linked to the post-encoding/consolidation memory period, one could argue that rTMS (or iTBS) could have modulated this phase by modifying excitability of contralateral (i.e., left) hemispheric structures via transcallosal pathways. In this sense, memory improvement following inhibitory rTMS of the right DLPFC could represent the counterpart of memory improvement recently reported in MCI following excitatory rTMS of the left parietal cortex (Manenti et al., 2011).

On the other hand, if the effects of TMS were linked to the retrieval phase, one could suppose an effect depending on modulation of excitability of right DLPFC and anatomically and functionally interconnected brain structures such as the posterior parietal cortex and middle temporal lobe (e.g., Simons and Spiers, 2003; Wagner et al., 2005; Vilberg and Rugg, 2008). Indeed, inhibition is postulated to be a mechanism by which right PFC exerts its effects on sub-cortical and posterior cortical regions to implement executive control (Aron et al., 2004). Direct proof of the existence of a top-down signal from the PFC to the temporal cortex and its contribution to the active retrieval process was provided by single-unit recordings from the infero-temporal cortex of posterior-split-brain monkeys (Tomita et al., 1999). In humans, DLPFC interacts with posterior cortical regions such middle temporal lobe during cognitive inhibition of unwanted memories. In particular, subjects submitted to the think/no think paradigm show higher right PFC activation when they have to voluntarily suppress memories for items previously presented. This right PFC activation corresponds to a decrement in hippocampal activity, suggesting that suppression of unwanted memories requires PFC recruitment to disengage hippocampal processing (Anderson et al., 2004). Inhibition of the right DLPFC could have interfered with this mechanism of memory suppression, thereby improving memory performance in the recognition phase.

Despite possible methodological bias comparing paper and pencil tests in MCI with computerized ones in HCs, a mechanism linked to disinhibition of posterior brain regions following inhibition of the right DLPFC could also explain enhanced memory performance in MCI patients. In fact, neuroimaging studies have revealed that the increased activation in right DLPFC is one of functional brain abnormalities associated with memory deficits in MCI and AD (e.g., Wang et al., 2006; Bai et al., 2009; Sperling et al., 2010). Usually, these findings have been interpreted as demonstrating recruitment of compensatory networks (e.g., Grady et al., 2003; Smith et al., 2007).

Partially in line with these evidences, the finding that the temporary inhibition of right DLPFC in MCI patients enhances memory performance suggests a cognitive benefit when there is reduced activity in this region during recognition memory tasks in patients with memory impairments. It may well be that the previously reported additional activation in DLPFC in MCI and $\mathrm{AD}$ patients, rather than reflecting the recruitment of cognitive resources to maintain task performance (e.g., Grady et al., 2003; Smith et al., 2007), represents a dysfunctional use of brain resources. Inhibitory rTMS over the right DLPFC may have the potential to modulate the activity in this dysfunctional network, enhancing function in healthy subjects or restoring an adaptive equilibrium in MCI patients (e.g., Fregni and Pascual-Leone, 2007).

However, the interpretation of the results of the MCI patients is limited due to the sample size. Future research is needed to replicate these findings.

In conclusion, our study reports an improvement in performance during recognition memory tasks in healthy subjects and in MCI patients with memory impairments following inhibitory rTMS over right DLPFC. These findings suggest that right DLPFC plays a pivotal role in the verbal and non-verbal recognition memory retrieval. Moreover, it demonstrates that rTMS may represent a promising therapeutic tool for memory impairments. 


\section{REFERENCES}

Anderson, M. C., Ochsner, K. N., Kuhl, B., Cooper, J., Robertson, E., Gabrieli, S. W., Glover, G. H., and Gabrieli, J. D. (2004). Neural systems underlying the suppression of unwanted memories. Science 303, 232-235.

Aron, A. R., Robbins, T. W., and Poldrack, R. A. (2004). Inhibition and the right inferior frontal cortex. Trends Cogn. Sci. 8, 170-177.

Bai, F., Zhang, Z., Watson, D. R., Yu, H., Shi, Y., Yuan, Y., Zang, Y., Zhu, C., and Qian, Y. (2009). Abnormal functional connectivity of hippocampus during episodic memory retrieval processing network in amnestic type mild cognitive impairment. Biol. Psychiatry 65, 951-958.

Buckner, R. L., and Wheeler, M. E. (2001). The cognitive neuroscience of remembering. Nat. Neurosci. Rev. 2, 624-634.

Cabeza, R. (2002). Hemispheric asymmetry reduction in older adults: the HAROLD model. Psychol. Aging 17, 85-100.

Cabeza, R., Anderson, N. D., Locantore, J. K., and McIntosh, A. R. (2002). Aging gracefully: compensatory brain activity in high-performing older adults. Neuroimage 17, 1394-1402.

Carlesimo, G. A., Buccione, I., Fadda, L., Graceffa, A., Mauri, M., Lo Russo, S., Bevilacqua, G., and Caltagirone, C. (2002). Standardizzazione di due test di memoria per uso clinico: Breve racconto e Figura di Rey. Nuova Rivista di Neurologia 12, 3-13.

Carlesimo, G. A., Caltagirone, C., and Gainotti, G. (1996). The Mental Deterioration Battery: normative data, diagnostic reliability and qualitative analyses of cognitive impairment. The group of the Standardization of the Mental Deterioration Battery. Eur. Neurol. 36, 378-384.

Cipolotti, L., Bird, C., Good, T., Macmanus, D., Rudge, P., and Shallice, T. (2006). Recollection and familiarity in dense hippocampal amnesia: a case study. Neuropsychologia 39, 151-172.

Cipolotti, L., Husain, M., Crinion, J., Bird, C. M., Khan, S. S., Losseff, N., Howard, R. S., and Leff, A. P. (2008). The role of the thalamus in amnesia: a tractography, high-resolution MRI and neuropsychological study. Neuropsychologia 46, 2745-2758.

Cotelli, M., Calabria, M., Manenti, R., Rosini, S., Maioli, C., Zanetti, O., and Miniussi, C. (2011). Brain stimulation improves associative memory in an individual with amnestic mild cognitive impairment. Neurocase Sep. 1. [Epub ahead of print]

Delis, D. C., Freeland, J., Kramer, J. H., and Kaplan, E. (1988). Integrating clinical assessment with cognitive neuroscience: construct validation of the California Verbal Learning Test. J. Consult. Clin. Psychol. 56, 123-130.

Di Lazzaro, V., Dileone, M., Pilato, F., Capone, F., Musumeci, G., Ranieri, F., Ricci, V., Bria, P., Di Iorio, R., de Waure, C., Pasqualetti, P., and Profice, P. (2011). Modulation of motor cortex neuronal networks by rTMS: comparison of local and remote effects of six different protocols of stimulation. J. Neurophysiol. 105, 2150-2156.

Fletcher, P. C., and Henson, R. N. A. (2001). Frontal lobes and human memory. Insights from functional neuroimaging. Brain 124, 849-881.

Floel, A., Poeppel, D., Buffalo, E. A., Braun, A.,Wu, C. W., Seo, H. J., Stefan, K., Knecht, S., and Cohen, L. G. (2004). Prefrontal cortex asymmetry for memory encoding of words and abstract shapes. Cereb. Cortex 14, 404-409.

Folstein, M. F., Folstein, S. E., and McHugh, P. R. (1975). Mini-mental state. A practical method for grading the cognitive state of patients for the clinician. J. Psychiatr. Res. 12, 189-198.

Fregni, F., and Pascual-Leone, A. (2007). Technology insight: noninvasive brain stimulation in neurology-perspectives on the therapeutic potential of rTMS and tDCS. Nat. Clin. Pract. Neurol. 3, 383-393.

Grady, C. L., McIntosh, A. R., Beig, S., and Craik, F. I. (2001). An examination of the effects of stimulus type, encoding task, and functional connectivity on the role of right prefrontal cortex in recognition memory. Neuroimage 14, 556-571.

Grady, C. L., McIntosh, A. R., Beig, S., Keightley, M. L., Burian, H., and Black, S. E. (2003). Evidence from functional neuroimaging of a compensatory prefrontal network in Alzheimer's disease. J. Neurosci. 23, 986-993.

Habib, R., Nyberg, L., and Tulving, E. (2003). Hemispheric asymmetries of memory: the HERA model revisited. Trends Cogn. Sci. 7, 241-245.

Huang, Y. Z., Edwards, M. J., Rounis, E., Bhatia, K. P., and Rothwell, J. C. (2005). Theta burst stimulation of the human motor cortex. Neuron 45, 201-206.
Hughes, C. P., Berg, L., Danziger, W. L., Coben, L. A., and Martin, R. L. (1982). A new clinical scale for the staging of dementia. Br. J. Psychiatry 140, 566-572.

Kirchhoff, B. A., Wagner, A. D., Maril, A., and Stern, C. E. (2000). Prefrontal-temporal circuitry for episodic encoding and subsequent memory. J. Neurosci. 20, 6173-6180.

Laudanna, A., Thornton, A. M., Brown, G., Burani, C., and Marconi, L. (1995). "Un corpus dell'italiano scritto contemporaneo dalla parte del ricevente," in Giornate Internazionali di Analisi Statistica dei Dati Testuali, 23 III, vol. 1, eds S. Bolasco, L. Lebart, and A. Salem (Roma, Cisu: Lawrence Erlbaum Associates), 103-109.

Manenti, R., Cotelli, M., Calabria, M., Maioli, C., and Miniussi, C. (2010). The role of the dorsolateral prefrontal cortex in retrieval from long-term memory depends on strategies: a repetitive transcranial magnetic stimulation study. Neuroscience 166, 501-507.

Manenti, R., Cotelli, M., and Miniussi, C. (2011). Successful physiological aging and episodic memory: a brain stimulation study. Behav. Brain Res. 216, 153-158.

McDermott, K. B., Buckner, R. L., Petersen, S. E., Kelley, W. M., and Sanders, A. L. (1999). Set- and codespecific activation in frontal cortex: an fMRI study of encoding and retrieval of faces and words. J. Cogn Neurosci. 11, 631-640.

Milner, B., Corsi, P., and Leonard, G. (1991). Frontal-lobe contribution to recency judgements. Neuropsychologia 29, 601-618.

Petersen, R. C., Smith, G. E., Waring, S. C., Ivnik, R. J., Tangalos, E. G., and Kokmen, E. (1999). Mild cognitive impairment, clinical characterization and outcome. Arch. Neurol. 56, 303-308.

Rosen, A. C., Prull, M. W., O’Hara, R., Race, E. A., Desmond, J. E., Glover G. H., Yesavage, J. A., and Gabrieli, J. D. (2002). Variable effects of aging on frontal lobe contributions to memory. Neuroreport 13, 2425-2428.

Rossi, S., Cappa, S. F., Babiloni, C., Pasqualetti, P., Miniussi, C. Carducci, F., Babiloni, F., and Rossini, P. M. (2001). Prefrontal cortex in long-term memory: an 'interference' approach using magnetic stimulation. Nat. Neurosci. 4, 948-952.

Rossi, S., Miniussi, C., Pasqualetti, P., Babiloni, C., Rossini, P. M., and Cappa, S. F. (2004). Age-related functional changes of prefrontal cortex in long-term memory: a repetitive transcranial magnetic stimulation study. J. Neurosci. 24, 7939-7944.

Rossini, P. M., Barker, A. T., Berardelli, A., Caramia, M. D., Caruso, G., Cracco, R. Q., Dimitrijević, M. R., Hallett, M., Katayama, Y., Lücking, C. H., Maertens de Noordhout, A. L., Marsden, C. D., Murray, N. M. F., Rothwell, J. C., Swash, M., and Tomberg, C. (1994). Noninvasive electrical and magnetic stimulation of the brain, spinal cord and roots: basic principles and procedures for routine clinical application. Report of an IFCN committee. Electroencephalogr. Clin. Neurophysiol. 91, 79-92.

Sandrini, M., Cappa, S. F., Rossi, S., Rossini, P. M., and Miniussi, C. (2003). The role of prefrontal cortex in verbal episodic memory: rTMS evidence. J. Cogn. Neurosci. 15, 855-861.

Simons, J. S., and Spiers, H. J. (2003). Prefrontal and medial temporal lobe interactions in long-term memory. Nat. Rev. Neurosci. 4, 637-648.

Smirni, D., Turriziani, P., Oliveri, M., Smirni, P., and Cipolotti, L. (2010). Standardizzazione di tre nuovi test di memoria di riconoscimento verbale e non verbale: uno studio preliminare. G. I. P. 1, 227-245.

Smith, G. E., Pankratz, V. S., Negasi, S., Machulda, M. M., Petersen, R. C., Boeve, B. F., Knopman, D. S., Lucas, J. A., Ferman, T. J., Graff-Radford, N., and Ivnik, R. J. (2007). A plateau in pre-Alzheimer memory decline: evidence for compensatory mechanisms? Neurology 69, 133-139.

Solé-Padullés, C., Bartrés-Faz, D., Junqué, C., Clemente, I. C., Molinuevo, J. L., Bargalló, N., Sánchez-Aldeguer, J., Bosch, B., Falcón, C., and Valls-Solé, J. (2006). Repetitive transcranial magnetic stimulation effects on brain function and cognition among elders with memory dysfunction. A randomized sham-controlled study. Cereb. Cortex 16, 1487-1493.

Spaniol, J., Davidson, P. S., Kim, A. S., Han, H., Moscovitch, M., and Grady, C. L. (2009). Event-related fMRI studies of episodic encoding and retrieval: meta-analyses using activation likelihood estimation. Neuropsychologia 47, 1765-1779.

Sperling, R. A., Dickerson, B. C., Pihlajamaki, M., Tannini, P., LaViolette, P. S., Vitolo, O. V., Hedden, T., Becker, J. A., Rentz, D. M., Selkoe, D. J., and Johnson, K. A. (2010). Functional alterations in memory networks in early 
Alzheimer's disease. Neuromolecular Med. 12, 27-43.

Squire, L. R. (2004). Memory systems of the brain: a brief history and current perspective. Neurobiol. Learn. Mem. 82, 171-177.

Tomita, H., Ohbayashi, M., Nakahara, K., Hasegawa, I., and Miyashita, Y. (1999). Top-down signal from prefrontal cortex in executive control of memory retrieval. Nature 401, 699-703.

Tulving, E., Kapur, S., Craik, F. I. M., Moscovitch, M., and Houle, S. (1994). Hemispheric encoding/retrieval asymmetry in episodic memory: positron emission tomography findings. Proc. Natl. Acad. Sci.U.S.A. 91, 2016-2020.

Turriziani, P., Oliveri, M., Salerno, S., Costanzo, F., Koch, G., Caltagirone, C., and Carlesimo, G. A. (2008). Recognition memory and prefrontal cortex: dissociating recollection and familiarity processes using rTMS. Behav. Neurol. 19, 23-27.
Turriziani, P., Smirni, D., Oliveri, M., Semenza, C., and Cipolotti, L. (2010). The role of the prefrontal cortex in familiarity and recollection processes during verbal and non-verbal recognition memory: an rTMS study. Neuroimage 52, 348-357.

van Kesteren, M. T., Fernández, G., Norris, D. G., and Hermans, E. J. (2010). Persistent schema-dependent hippocampalneocortical connectivity during memory encoding and postencoding rest in humans. Proc. Natl. Acad. Sci. U.S.A. 107, 7550-7555.

Vilberg, K., and Rugg, M. D. (2008). Memory retrieval and the parietal cortex: a review of evidence from a dual-process perspective. Neuropsychologia 46, 1787-1799.

Wagner, A. D., Poldrack, R. A., Eldridge, L. L., Desmond, J. E., Glover, G. H., and Gabrieli, J. D. (1998). Material-specific lateralization of prefrontal activation during episodic encoding and retrieval. Neuroreport 9, 3711-3717.

Wagner, A. D., Shannon, B. J., Kahn, I., and Buckner, R. L. (2005). Parietal lobe contributions to episodic memory retrieval. Trends Cogn. Sci. 9, 445-453.

Wang, L., Zang, Y., He, Y., Liang, M., Zhang, X., Tian, L., Wu, T., Jiang, T., and Li, K. (2006). Changes in hippocampal connectivity in the early stages of Alzheimer's disease: evidence from resting state fMRI. Neuroimage 31, 496-504.

Warrington, E. K. (1984). Recognition Memory Test. Windsor, Berks: NFER-Nelson.

Warrington, E. K. (1996). The Camden Memory Tests. Hove, UK: Psychology Press.

Conflict of Interest Statement: The authors declare that the research was conducted in the absence of any commercial or financial relationships that could be construed as a potential conflict of interest.

Received: 30 November 2011; accepted: 10 March 2012; published online: 10 April 2012.

Citation: Turriziani $P$, Smirni D, Zappalà G, Mangano GR, Oliveri $M$ and Cipolotti L (2012) Enhancing memory performance with rTMS in healthy subjects and individuals with Mild Cognitive Impairment: the role of the right dorsolateral prefrontal cortex. Front. Hum. Neurosci. 6:62. doi: 10.3389/fnhum.2012.00062

Copyright (C) 2012 Turriziani, Smirni, Zappalà, Mangano, Oliveri and Cipolotti. This is an open-access article distributed under the terms of the Creative Commons Attribution Non Commercial License, which permits non-commercial use, distribution, and reproduction in other forums, provided the original authors and source are credited. 